\title{
Atomistic Calculations of Heat Transport in a Silicon Crystal ${ }^{*}$
}

\author{
Nobuhiko Kobayashi ${ }^{\dagger}$ and Kohei Yamamoto \\ Institute of Applied Physics and Tsukuba Research Center for Interdisciplinary Materials Science, \\ University of Tsukuba, Tsukuba, Ibaraki 305-8573, Japan \\ Hiroyuki Ishii \\ Institute of Applied Physics and Tsukuba Research Center for Interdisciplinary Materials Science, \\ University of Tsukuba, Tsukuba, Ibaraki 305-8573, Japan, and \\ PRESTO, Japan Science and Technology (JST) Agency, Kawaguchi, Saitama 332-0012, Japan \\ Kenji Hirose \\ Green Innovation Research Laboratories, NEC Corporation, Tsukuba, Ibaraki 305-8501, Japan
}

(Received 13 January 2014; Accepted 6 March 2014; Published 5 April 2014)

\begin{abstract}
We have performed atomistic calculations of phonon transport properties. The dynamical matrix is obtained from ab initio calculations, and phonon transport properties are analyzed by the nonequilibrium Green's function method. As an example of the applications of the method, we present the temperature dependence of heat transport properties and the phonon lifetime due to phonon-phonon scatterings by the anharmonic terms of interatomic forces for a silicon crystal. [DOI: 10.1380/ejssnt.2014.154]
\end{abstract}

Keywords: Density functional calculations; Green's function methods; Phonons; Silicon

\section{INTRODUCTION}

Heat transport of nanoscale systems has attracted considerable attention for applications in nanometer-scale electron devices and thermoelectric devices. Heat transport properties are sensitive to atomic-scale crystal structures, and thus, the development of theoretical approaches from atomistic levels is indispensable. In semiconductor and insulator materials, heat energy is transported primarily through lattice vibrations, i.e., phonons, and phonon thermal transport has been studied by various theoretical methods such as molecular dynamics [1-5],

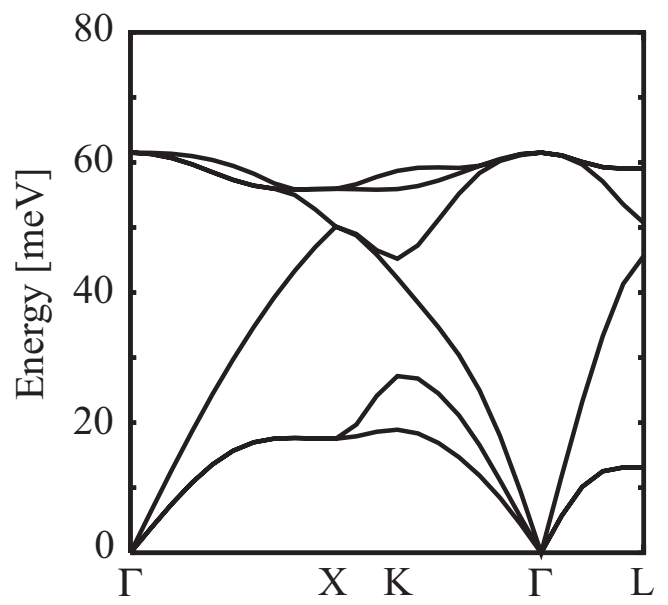

FIG. 1: Phonon dispersion of silicon crystal calculated using dynamical matrix from ab initio calculations.

\footnotetext{
*This paper was presented at the 12 th International Conference on Atomically Controlled Surfaces, Interfaces and Nanostructures (ACSIN-12) in conjunction with the 21st International Colloquium on Scanning Probe Microscopy (ICSPM21), Tsukuba International Congress Center, Tsukuba, Japan, November 4-8, 2013.

${ }^{\dagger}$ Corresponding author: nkoba@bk.tsukuba.ac.jp
}

Boltzmann equation method [6, 7], and nonequilibrium Green's function method [8-12].

In this paper, we report on atomistic calculations of phonon transport properties using ab initio calculations and the nonequilibrium Green's function method. The dynamical matrix is calculated on the basis of the density functional theory, and the transmission function is obtained using the Green's function, which is useful for the analysis of atomic-scale structures and the inclusion of quantum effects. Furthermore, phonon lifetime due to the phonon-phonon scatterings by the anharmonic terms of interatomic forces is presented and discussed.

\section{METHOD}

We take the Hamiltonian $H$ of the system for phonon transport as

$$
H=\sum_{\substack{i \in s y s \\ \alpha=x, y, z}} \frac{1}{2 M_{i}} p_{i \alpha}^{2}(t)+\frac{1}{2} \sum_{\substack{i, j \in s y s \\ \alpha, \beta=x, y, z}} u_{i \alpha}(t) K_{i \alpha, j \beta} u_{j \beta}(t) .
$$

$M_{i}$ is the mass of atom $i$ and $u_{i \alpha}(t)$ is an operator for the displacement of atom $i$ along the $\alpha$ direction from the equilibrium position. The total Hamiltonian is split into four parts: $H=H_{L}+H_{S}+H_{R}+H_{\text {int }}$, where $H_{L(R)}$ is the Hamiltonian for the left (right) lead, $H_{S}$ is for the scattering region, and $H_{\text {int }}$ is for the interaction between the scattering region and the left (right) lead. The thermal current $J_{t h}$ and thermal conductance $G(T)$ from the left lead to the scattering region are expressed as

$$
\begin{aligned}
J_{t h} & =-\left\langle\dot{H}_{L}\right\rangle \\
& =\int_{0}^{\infty} \frac{d \omega}{2 \pi} \hbar \omega\left[n\left(\omega, T_{L}\right)-n\left(\omega, T_{R}\right)\right] \zeta(\omega) \\
G(T) & =\frac{k_{B}}{2 \pi} \int_{0}^{\infty} d \omega \frac{\hbar \omega}{k_{B}} \frac{\partial n}{\partial T} \zeta(\omega) .
\end{aligned}
$$

Here, the bracket $\langle\cdots\rangle$ denotes the nonequilibrium statistical average of the physical observable, and $n\left(\omega, T_{L(R)}\right)$ 


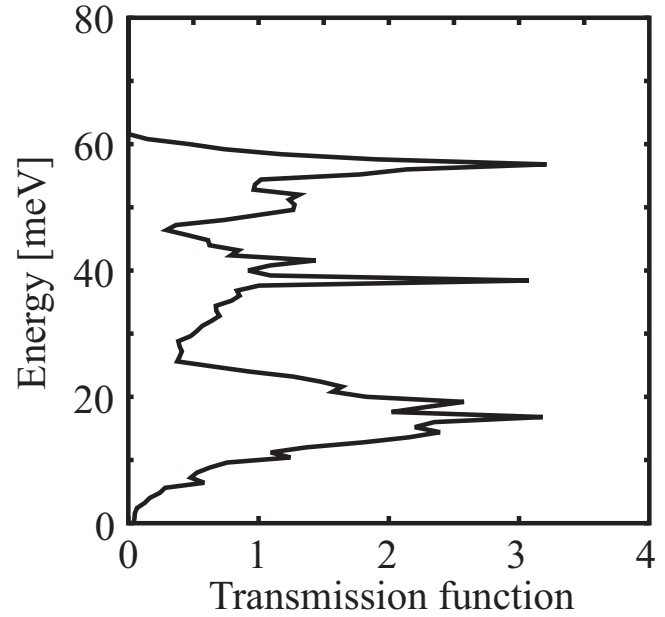

FIG. 2: Transmission function for silicon crystal.

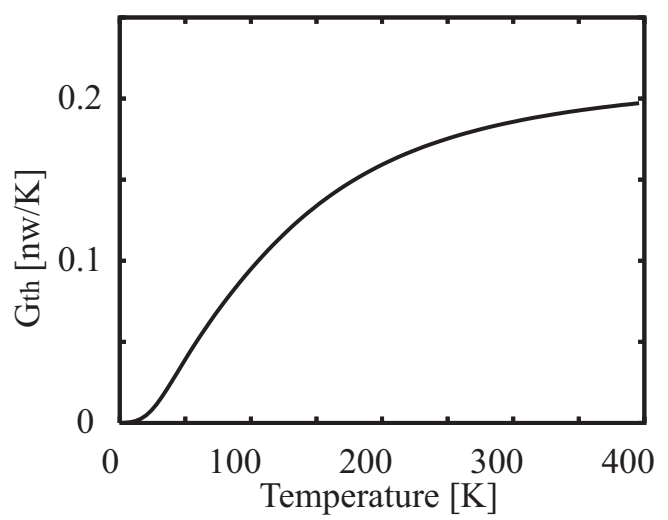

FIG. 3: Temperature dependence of heat conductance of silicon crystal.

is the Bose-Einstein distribution function of equilibrium phonons with an energy of $\hbar \omega$ in the left (right) lead at temperature $T$. The transmission function $\zeta(\omega)$ is expressed using the retarded (advanced) Green's function $G^{r(a)}(\omega)$ and the coupling constant $\Gamma_{L(R)}(\omega)$ as

$$
\zeta(\omega)=\operatorname{Tr}\left[\Gamma_{L}(\omega) G^{r}(\omega) \Gamma_{R}(\omega) G^{a}(\omega)\right] .
$$

The retarded (advanced) Green's function for the scattering region is calculated as

$$
G^{r(a)}(\omega)=\left[\omega^{2} M-K-\Sigma_{L}^{r(a)}-\Sigma_{R}^{r(a)}\right]^{-1},
$$

where $M$ is the diagonal matrix whose element is the mass of an atom and $\Sigma_{L(R)}^{r(a)}(\omega)$ is the retarded (advanced) selfenergy due to the coupling to the left (right) semi-infinite lead with the scattering region. The dynamical matrix $K$ is constructed from ab initio calculations [13]. To confirm the validity of the present method, we show in Fig. 1 the phonon dispersion relations calculated using the dynamical matrix $K$, which is in good agreement with the experimental data [14].

\section{RESULTS}

Figures 2 and 3 show the transmission function integrated in the Brillouin zone and the temperature depen-

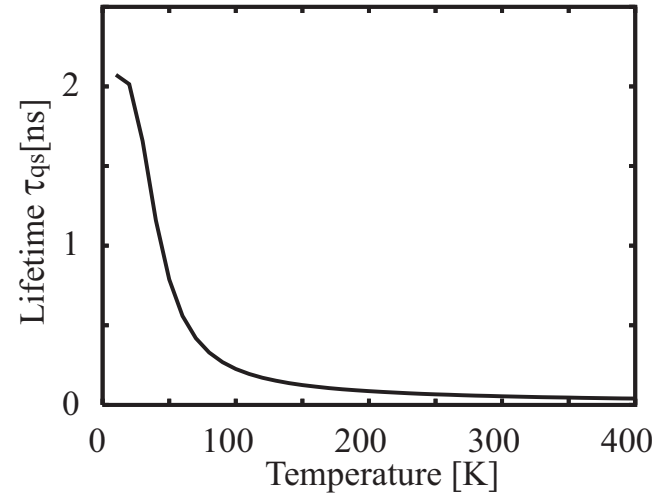

FIG. 4: Temperature dependence of lifetime of TA phonon at $\mathrm{X}$ point.

dence of the heat conductance of a silicon crystal, respectively, where the heat conductance per face of a unit cell for the fcc lattice in the [100] direction is shown. At lowenergy regimes, a small number of excited phonons contribute to the heat transport, and the transmission function and conductance are thus small. As temperature increases, various phonon modes begin to contribute to the transport and the conductance increases. Since the phonon energy has an upper limit, as shown in Fig. 1, the transmission function becomes zero for high energy regimes and the conductance saturates at a high temperature.

On the other hand, as temperature increases, phononphonon scatterings become enhanced and the conductance should show a decrease at the high-temperature regime. For bulk silicon, the scattering effect is seen above $30 \mathrm{~K}$ [15]. To discuss this effect, we investigate the phonon-phonon interaction. Note that, in the above calculations, phonons are treated as noninteracting with an infinite lifetime in the harmonic approximation. Here, we deal with the anharmonic terms of the interatomic force to analyze the phonon-phonon scatterings.

Force $F_{i}$ acting on an atom $i$ is expanded using the displacement $x_{j}$ of an atom $j$ as [16]

$$
F_{i}=\sum_{j} \Psi_{i, j} \cdot x_{j}-\frac{1}{2} \sum_{j, k} \Phi_{i, j, k} \cdot x_{j} x_{k} .
$$

We obtained the third term of the potential $\Phi_{i, j, k}$ using the Lennard-Jones potential for simplicity in this work. It is possible to calculate the third and higher terms using ab initio calculations in the same manner. The phonon lifetime $\tau_{q s}$ is calculated as [17]

$$
\begin{aligned}
& \frac{1}{\tau_{q s}}=\pi \sum_{q^{\prime} s^{\prime}} \sum_{s^{\prime \prime}}\left|V_{3}\left(q s, q^{\prime} s^{\prime}, q^{\prime \prime} s^{\prime \prime}\right)\right|^{2} \\
& {\left[\left(n_{q^{\prime} s^{\prime}}+n_{q^{\prime \prime} s^{\prime \prime}}+1\right) \delta\left(\omega(q s)-\omega\left(q^{\prime} s^{\prime}\right)-\omega\left(q^{\prime \prime} s^{\prime \prime}\right)\right)\right.} \\
& \left.+2\left(n_{q^{\prime} s^{\prime}}-n_{q^{\prime \prime} s^{\prime \prime}}\right) \delta\left(\omega(q s)+\omega\left(q^{\prime} s^{\prime}\right)-\omega\left(q^{\prime \prime} s^{\prime \prime}\right)\right)\right],
\end{aligned}
$$

where $q$ is a phonon wave vector and $s$ is a phonon branch.

Figure 4 shows the temperature dependence of phonon lifetime for the transverse acoustic phonon mode. It is calculated using the primitive cell for a periodic system. With increasing temperature, the phonon lifetime becomes short, which causes the decrease in thermal conductance at a high temperature. Note that the calculation 
of the phonon lifetime enables us to analyze the transport properties using the nonequilibrium Green's function, including the effects of phonon-phonon scatterings. The self-energy for phonon-phonon scatterings is obtained using the Kramers-Kronig relation with coupling constant, and then the conductance is analyzed, including the anharmonic effects.

\section{SUMMARY}

In summary, we reported the calculations of heat transport using ab initio calculations and the Green's function method and phonon lifetime including anharmonic effects from atomistic viewpoints. As an example of the method, we show the temperature dependences of the conductance and phonon lifetime for a bulk silicon crystal. The present theoretical approach is useful for the analysis of not only heat transport but also thermoelectric properties by the combination with electron transport calculations.

\section{Acknowledgments}

This work was supported by the Grant-in-Aid for Scientific Research from the Ministry of Education, Culture, Sports, Science and Technology, Japan. Numerical calculations were in part performed at the Supercomputer Center, ISSP, University of Tokyo.
[1] D. J. Evans, Phys. Lett. A 91, 457 (1982).

[2] A. S. Henry and G. Chen, J. Comput. Theor. Nanosci. 5, 141 (2008).

[3] J. V. Goicochea, M. Madrid, and C. Amon, ASME J. Heat Transfer 132, 012401 (2010).

[4] K. Esfarjani, G. Chen, and H. T. Stokes, Phys. Rev. B 84, 085204 (2011).

[5] T. Hori, T. Shiga, and J. Shiomi, J. Appl. Phys. 113, 203514 (2013)

[6] A. J. H. McGaughey and M. Kaviany, Phys. Rev. B 69, 094303 (2004).

[7] D. A. Broido, A. Ward, and N. Mingo, Phys. Rev. B 72, 014308 (2005).

[8] A. Ozpineci and S. Ciraci, Phys. Rev. B 63, 125415 (2001).
[9] N. Mingo and L. Yang, Phys. Rev. B 68, 245406 (2003).

[10] T. Yamamoto and K. Watanabe, Phys. Rev. Lett. 96, 255503 (2006).

[11] T. Markussen, A. P. Jauho, and M. Brandbyge, Phys. Rev. B 79, 035415 (2009).

[12] K. Yamamoto, H. Ishii, N. Kobayashi, and K. Hirose, Appl. Phys. Express 4, 085001 (2011).

[13] http://www.quantum-espresso.org/

[14] G. Nilsson and G. Nelin, Phys. Rev. B 6, 3777 (1972).

[15] C. J. Glassbrenner and G. A. Slack, Phys. Rev. 134, A1058 (1964).

[16] K. Esfarjani and H. T. Stokes, Phys. Rev. B 77, 144112 (2008).

[17] A. Debernardi, Phys. Rev. B 57, 12847 (1998). 